\title{
Carathéodory's Principle and the Existence of Global Integrating Factors
}

\author{
J. B. Boyling \\ Department of Applied Mathematic and Theoretical Physics \\ University of Cambridge
}

Received May 8, 1968

\begin{abstract}
A proof is given of a theorem on the integrability of Pfaffian forms which is used in Carathéodory's approach to thermodynamics. It is pointed out that Carathéodory's original proof of the existence of entropy and of absolute temperature is incomplete, since it fails to take into account the local nature of this theorem. By combining the theorem with the results of BUCHDAHL and Greve on the existence of continuous empirical entropy functions, it is shown that the First and Second Laws of Thermodynamics imply the existence of a globally defined differentiable empirical entropy function for every simple thermodynamic system. This result supplies the missing step in Carathéodory's argument and makes a separate proof of the principle of increase of entropy unnecessary.
\end{abstract}

\section{Introduction}

It has been pointed out by Bernsters [1] that Carathéodory's proof [2] of the existence of entropy and of absolute temperature is incomplete, since the local nature of a certain theorem on the integrability of Pfaffians is not fully taken into account. The theorem in question $[3,4]$ runs as follows:

Theorem. Let $M$ be a $C^{\infty}$ differentiable manifold [5] (finite-dimensional and without boundary), $\psi$ an everywhere non-vanishing $C^{\infty}$ differential 1-form on $M$. Then the following three conditions are equivalent:

(i) Given $x$ in $M$, there exists an open neighbourhood $V$ of $x$ in $M$ such that each neighbourhood $W$ of $x$ in $V$ contains a point $y$ which cannot be connected to $x$ by a piece-wise $C^{\infty}$ path $\gamma$ in $V$ which satisfies $\psi\{\dot{\gamma}(t)\}=0$ whenever $\dot{\gamma}(t)$ is defined.

(ii) $\psi \wedge d \psi=0$.

(iii) Given $x$ in $M$, there exists an open neighbourhood $V$ of $x$ in $M$ such that the restriction $\psi \mid V$ of $\psi$ to $V$ is of the form $\psi \mid V=f d g$, where $f$ and $g$ are $C^{\infty}$ functions on $V$.

For the sake of completeness, a proof of this theorem is given in the appendix.

Carathéodory's form of the Second Law of Thermodynamics (Carathéodory's Principle) implies that a certain everywhere non-vanishing differential 1-form $\psi$ on the thermodynamic configuration space $M$ of a 
simple [2] thermodynamic system satisfies condition (i) above. It therefore satisfies condition (iii). However, in the absence of further information, there is no reason to suppose that it will satisfy the stronger condition:

(iii)' There exist $C^{\infty}$ functions $f$ and $g$ on $M$ such that $\psi=f d g$. Thus, although $\psi$ has a local integrating factor in the neighbourhood of each point of $M$, it need not have a global integrating factor.

It might be thought that (i) would imply (iii)' if the manifold $M$ were topologically sufficiently simple, e. g. simply-connected or contractible. That this is not the case is made evident by the following simple example, in which $M$ is contractible.

Let $M$ be the plane $\mathbb{R}^{2}$, and let $\psi$ be the differential 1 -form

$$
\psi=y^{3}(1-y)^{2} d x+\left\{y^{3}-2(1-y)^{2}\right\} d y .
$$

Then $\psi$ is everywhere non-zero and satisfies (i). However (iii)' cannot hold. For suppose $\psi=f d g$, where $f$ and $g$ are $C^{\infty}$ functions on the plane. Then, on the infinite strip $0<y<1, g$ must be of the form

$$
g(x, y)=h\left(x+\frac{1}{y^{2}}+\frac{1}{1-y}\right)
$$

where $h$ is a $C^{\infty}$ function of a single real variable. The function $f$ is therefore given on this strip by

$$
f(x, y) h^{\prime}\left(x+\frac{1}{y^{2}}+\frac{1}{1-y}\right)=y^{3}(1-y)^{2} .
$$

Since $f$ is continuous and everywhere non-zero, we have

and

$$
f(x, y) \rightarrow f(x, 0) \neq 0 \text { as } y \rightarrow 0 \text { from above }
$$

$$
f(x, y) \rightarrow f(x, 1) \neq 0 \quad \text { as } \quad y \rightarrow 1 \text { from below . }
$$

Consequently $t^{3 / 2} h^{\prime}(t)$ and $t^{2} h^{\prime}(t)$ must both tend to (finite) non-zero limits as $t \rightarrow+\infty$, and this is impossible.

BeRnsteIn shows [1] that the existence of a global integrating factor is not in fact essential to Carathéodory's argument; the above-mentioned local theorem is sufficient by itself. Assuming the First and Second Laws of Thermodynamics and the existence of an empirical temperature scale (Zeroth Law), he obtains an everywhere non-vanishing function $L$ of temperature only, determined to within a non-zero multiplicative constant, such that $L^{-1} \psi$ is a closed differential 1-form on $M$ for each simple system. When suitably normalized, this function $L$ is the absolute temperature. The systems considered by Bernstein have thermodynamic configuration spaces $M$ of a very simple kind. In particular, they satisfy the condition

$$
H^{1}(M ; \mathbb{R})=0
$$


where $H^{1}(M ; \mathbb{R})$ is the first cohomology group [5] of $M$ with real coefficients. For such systems $L^{-1} \psi=d S$, where the differentiable function $S$ on $M$, determined to within an additive constant, is the entropy of the system.

We wish to consider a more general situation, in which $M$ is an arbitrary (paracompact) connected, finite-dimensional $C^{\infty}$ differentiable manifold without boundary [5], not necessarily satisfying the condition

$$
H^{1}(M ; \mathbb{R})=0 .
$$

In justification of this greater degree of generality, we remark that mechanical systems are known whose configuration spaces do not satisfy the above condition. A simple example of such a system is that of a rigid body constrained to rotate about a fixed axis. Mechanical systems of this kind would be expected to have thermodynamic analogues whose configuration spaces $M$ would also fail to satisfy the condition

$$
H^{1}(M ; \mathbb{R})=0 .
$$

For such systems, Bernstein's argument would not demonstrate the existence of an entropy function, since the closed form $L^{-1} \psi$ need not be exact. A different kind of argument is therefore necessary in such cases.

A completely different approach to the problem is that of BUCHDAHL and GREve [6], who show under rather general conditions that the Second Law of Thermodynamics implies the existence of a continuous empirical entropy function on $M$, i. e. a continuous real-valued function on $M$ which satisfies the principle of increase of entropy for adiabatic transitions. Actually it is not even necessary to assume the full content of the Second Law to obtain such a function, as LANDSBERG [7] has pointed out. The difficulty with this approach is that the continuous empirical entropy function obtained is not necessarily differentiable, nor is there any obvious way of modifying it to make it so. Indeed, its level surfaces need not be submanifolds of $M$. It cannot therefore be used directly in the search for a global integrating factor for $\psi$.

We shall show that the existence of a continuous empirical entropy and the existence of local integrating factors for $\psi$ together imply the existence of a global integrating factor converting $\psi$ into the differential of a (differentiable) empirical entropy on $M$. Our argument makes no use of the concept of temperature. However, if an empirical temperature scale is assumed to exist, our result provides the necessary starting point for the usual arguments leading to the existence of entropy proper and of the absolute temperature scale. Since these arguments are quite standard $[2,3,4]$, they will not be repeated here. An advantage of 
this approach is that no separate proof of the principle of increase of entropy is required [3], since the true entropy is a strictly increasing function of the empirical entropy obtained here.

In section 2 we demonstrate the existence of a continuous (global) empirical entropy $\sigma$ by methods similar to those of BUCHDAHL and GREve [6], but without assuming the Second Law of Thermodynamics. The First and Second Laws are introduced in section 3 together with certain supplementary smoothness assumptions, and it is shown that a differentiable local empirical entropy can be defined in the neighbourhood of each point of $M$. The construction of a differentiable global empirical entropy $s$ is finally accomplished in section 4 .

\section{The Construction of a Continuous Global Empirical Entropy}

Throughout this paper we shall focus our attention on a single thermodynamic system $K$, which we shall suppose to contain no internal adiabatic partitions and to be free from hysteresis. This is slightly more general than a simple system in the sense of CARATHÉodory [2] or a standard system in the sende of BuchdaHL [3].

In this section we shall suppose that the possible (equilibrium) states of $K$ may be represented by the points of a connected separable topological space [8] $M$.

By an adiabatic transition of $K$ we shall mean a (not necessarily reversible or quasi-static) transition of $K$ in which the interaction between $K$ and its surroundings is purely mechanical, in the wide sense in which (for example) electromagnetic and gravitational interactions are counted as being mechanical.

A preorder [9] relation $\leqq$ on $M$ may now be defined as follows. We write $x \leqq y$ (or $y \geqq x$ ) and say the state $y$ is accessible from the state $x$, when the system $K$ is capable of undergoing an adiabatic transition from the state $x$ to the state $y$. When this is not the case, we write $x>y$ (or $y<x$ ) and say that $y$ is inaccessible from $x$. It is clear from its mode of definition that the relation $\leqq$ is a preorder relation, i. e. that it is reflexive

and transitive

$$
x \leqq x
$$

$$
x \leqq y \text { and } y \leqq z \Rightarrow x \leqq z .
$$

The preorder relation $\leqq$ induces an equivalemce relation $\sim$ on $M$ defined by

$$
x \sim y \text { if and only if } x \leqq y \text { and } y \leqq x .
$$

When $x \sim y$, we shall say that the states $x$ and $y$ are mutually accessible. The equivalence classes of points of $M$ under $\sim$ will be called mutual accessibility classes. Let $\Sigma$ be the set of all mutual accessibility classes 
and let $\pi: M \rightarrow \Sigma$ be the natural projection which associates with a point $x$ of $M$ the unique mutual accessibility class $\pi(x)$ to which it belongs. Then $\leqq$ passes to the quotient to induce an order [9] relation on $\Sigma$, which we shall, by abuse of language, also denote by $\leqq$.This order relation may be unambiguously defined by

$$
\pi(x) \leqq \pi(y) \text { if and only if } x \leqq y .
$$

We shall suppose that the preorder relation $\leqq$ on $M$ satisfies the following four conditions:

(A) Given $x$ and $y$ in $M$, then either $x \leqq y$ or $y \leqq x$.

(B) If $x, y \in M$ and $x<y$, then there is a neighbourhood $V$ of $x$ and a neighbourhood $W$ of $y$ such that

$$
x^{\prime} \in V \text { and } y^{\prime} \in W \Rightarrow x^{\prime}<y^{\prime} .
$$

(C) Given $x$ in $M$, there exists a $y$ in $M$ such that $x<y$.

(D) Given $x$ in $M$, there exists a $y$ in $M$ such that $y<x$.

Condition (A), which is physically reasonable for systems of the type under consideration, ensures that $\Sigma$ is totally ordered under $\leqq$. Condition (B) is a continuity assumption linking the preorder relation $\leqq$ with the topology of $M$. It states that the preorder relation $\leqq$ is closed [9], i. e. that its graph

$$
\{(x, y) \in M \times M ; x \leqq y\}
$$

is a closed subset of the topological product $M \times M$. Condition (C) states that $M$ has no maximal element under $\leqq$ and implies that the totally ordered set $\Sigma$ has no greatest element. Similarly, condition (D) states that $M$ has no minimal element and implies that $\Sigma$ has no smallest element. In particular, we see that $\Sigma$ must be an infinite set. Conditions (C) and (D) have the physical consequence that $K$ has no state of maximum entropy and no state of minimum entropy. We remark that, although the Third Law of Thermodynamics imposes a lower bound on the possible values of the entropy of $K$, this lower bound is never attained, since the absolute zero of temperature can never be reached.

By a global empirical entropy $\sigma$ on $M$ we shall mean a real-valued function $\sigma$ on $M$ such that

$$
x \leqq y \quad \text { if and only if } \sigma(x) \leqq \sigma(y) .
$$

By a local empirical entropy $\sigma_{V}$ on a subset $V$ of $M$ we shall mean a real-valued function $\sigma_{V}$ on $V$ such that, for states $x$ and $y$ in $V$,

$$
x \leqq y \quad \text { if and only if } \sigma_{V}(x) \leqq \sigma_{V}(y) .
$$

We shall now show from postulates (A), (B), (C) and (D) that there exists a continuous global empirical entropy $\sigma$ on $M$. 
Let $\tau$ be the order topology [8] on $\Sigma$, i. e. the weakest topology on $\Sigma$ for which the order relation $\leqq$ is closed, and let $\tau^{\prime}$ be the quotient topology on $\Sigma$, i. e. the strongest topology on $\Sigma$ for which the natural projection $\pi: M \rightarrow \Sigma$ is continuous. It follows at once from (B) that $\tau$ is weaker than $\tau^{\prime}$ (in the wide sense), i. e. that the identity map

$$
\iota:\left(\Sigma, \tau^{\prime}\right) \rightarrow(\Sigma, \tau)
$$

is continuous. Thus $(\Sigma, \tau)$ is the image of a connected separable space $M$ under a continuous map $\iota \circ \pi$. It follows that $(\Sigma, \tau)$ is itself connected and separable. Since $\Sigma$ is an infinite totally ordered set with no greatest element and no smallest element, and since $\tau$ is its order topology, it follows [see Ref. (8), p. 118] that there exists an order-preserving homeomorphism $h$ of $(\Sigma, \tau)$ onto an open interval of the real line, which we may take to be the semi-infinite open interval $(0, \infty)$. Defining

$$
\sigma=h \circ \iota \circ \pi: M \rightarrow(0, \infty)
$$

we see that $\sigma$ is a continuous global empirical entropy on $M$.

\section{The Existence of Differentiable Local Empirical Entropies}

From now on we shall assume that the connected separable space $M$ is a finite-dimensional $C^{\infty}$ differentiable manifold without boundary [5]. In this section we shall deduce from the First and Second Laws of Thermodynamics and certain auxiliary assumptions that there is an open covering $\mathscr{V}$ of $M$ on each set $V$ of which a $C^{\infty}$ local empirical entropy $s_{V}$ is defined whose differential never vanishes. In particular, the mutual accessibility classes are $C^{\infty}$ submanifolds of $M$ of codimension 1 .

A transition of the system $K$ will be said to be quasi-static if it proceeds so slowly that $K$ can be considered to be in thermodynamic equilibrium at each stage of the process. The transition can then be represented by the motion of a point in $M$. In fact we shall suppose that every quasi-static transition of $K$ may be represented by a piecewise $C^{\infty}$ path $\gamma:[0,1] \rightarrow M$.

Instead of assuming the First Law of Thermodynamics directly, we shall suppose that the following consequence of it holds:

(E) There exists an everywhere non-vanishing $C^{\infty}$ differential 1-form $\psi$ on $M$ with the property that a piece-wise $C^{\infty}$ path $\gamma$ in $M$ represents a possible quasi-static adiabatic transition of $K$ if and only if $\psi\{\dot{\gamma}(t)\}=0$ whenever the tangent vector $\dot{\gamma}(t)$ to the path exists.

Before proceeding any further, we shall indicate briefly how (E) might be deduced from the First Law.

It is an immediate consequence [3, 4] of the First Law and postulate (A) of section 2 that there exists a real-valued function $U$ on $M$ with the property that the work done by $K$ on its surroundings in the course 
of any adiabatic transition (quasi-static or otherwise) is equal to the amount by which $U$ decreases in the transition. The function $U$ on $M$ is determined to within an additive constant and is called the internal energy of the system $K$. Assuming that $U$ is a $C^{\infty}$ function on $M$ and that there exists a $C^{\infty}$ differential 1 -form $\omega$ on $M$ with the property that the work done by $K$ on its surroundings in the course of any quasi-static transition (adiabatic or otherwise) is given by

$$
\int_{\gamma} \omega=\int_{0}^{1} \omega\{\dot{\gamma}(t)\} d t
$$

we see that every quasi-static adiabatic transition $\gamma$ must satisfy the condition

where

$$
\psi\{\dot{\gamma}(t)\}=0 \text { whenever } \dot{\gamma}(t) \text { exists },
$$

$$
\psi=\omega+d U .
$$

Assuming that $\psi$ never vanishes and that every piece-wise $C^{\infty}$ path $\gamma$ in $M$ satisfying the above condition represents a possible quasi-static transition of $K$, we recover condition (E).

The Second Law of Thermodynamics will be assumed in the form of Carathéodory's Principle:

$\left(\mathrm{D}^{\prime}\right)$ Given a point $x$ of $M$ and a neighbourhood $V$ of $x$ in $M$, there exists a point $y$ in $V$ such that $y<x$.

Since $\left(\mathrm{D}^{\prime}\right)$ implies (D), postulate (D) is now redundant. It follows from $\left(\mathrm{D}^{\prime}\right)$ that every point $x$ of $M$ must satisfy one or other of the following two mutually exclusive conditions:

(i) Every neighbourhood of $x$ contains points $y$ such that $y<x$ and points $z$ such that $z>x$.

(ii) $x$ has a neighbourhood consisting entirely of points $y$ satisfying $y \leqq x$.

If (i) holds, the point $x$ will be said to be of type (i); if (ii) holds, $x$ will be said to be of type (ii).

For technical reasons we shall need one further assumption:

(F) The mutual accessibility classes are connected subsets of $M$.

We note first, as an immediate consequence of $\left(\mathrm{D}^{\prime}\right)$ and $(\mathrm{E})$, that the everywhere non-vanishing $C^{\infty}$ differential 1-form $\psi$ on $M$ satisfies condition (i) of the theorem on Pfaffians quoted in section 1. It therefore satisfies condition (iii). Thus, given $x$ in $M$, there exists an open neighbourhood $V$ of $x$ in $M$ such that

$$
\psi \mid V=\lambda_{V} d s_{V}
$$

where $\lambda_{V}$ and $s_{V}$ are $C^{\infty}$ functions on $V$ and $s_{V}(x)=0$.

Since $d s_{V}$ is everywhere non-zero on $V$, we may assume without loss of generality (by making $V$ smaller and altering $s_{V}$ by a constant scale 
factor if necessary) that a local coordinate system $\left(x_{1}, \ldots, x_{n}\right)$ for $M$ is defined on $V$, that $x_{n}=s_{V}$, and that the points of $V$ are represented in this local coordinate system by the points of the open ball

$$
x_{1}^{2}+x_{2}^{2}+\cdots+x_{n}^{2}<1
$$

whose centre corresponds to the point $x$.

If $y$ and $z$ are points of $V$ such that $s_{V}(y)=s_{V}(z)$, then clearly $y$ and $z$ may be connected by a $C^{\infty}$ path $\gamma$ in $V$ on which $s_{V}$ is constant, so that $\psi(\dot{\gamma})$ vanishes identically on $\gamma$. It follows from $(E)$ that this path and its reverse both correspond to possible quasi-static adiabatic transitions of $K$. Hence $y \sim z$ and so $\sigma(y)=\sigma(z)$. Thus $\sigma(y)=f\left\{s_{V}(y)\right\}$ for $y$ in $V$, where $f$ is a continuous real-valued function on the open interval $(-1,1)$.

Now it follows from $\left(\mathrm{D}^{\prime}\right)$ that the function $f$ can have no local minimum, i.e. there exists no point $s_{0}$ in $(-1,1)$ such that $f(s) \geqq f\left(s_{0}\right)$ for all $s$ in some neighbourhood $\left(s_{0}-\delta, s_{0}+\delta\right)$ of $s_{0}$ in $(-1,1)$. For suppose that such an $s_{0}$ exists, and let $x_{0}$ be any point of $V$ for which $s_{V}\left(x_{0}\right)=s_{0}$. Then every point of the neighbourhood $s_{V}^{-1}\left(s_{0}-\delta, s_{0}+\delta\right)$ of $x_{0}$ is accessible from $x_{0}$, contradicting $\left(\mathrm{D}^{\prime}\right)$.

Since $f$ has no local minimum, it follows that either

(a) $f$ is strictly monotonic

or

(b) $f$ has a maximum at a point $\xi$ of $(-1,1)$, increases strictly in $(-1, \xi]$ and decreases strictly in $[\xi, 1)$.

For suppose (a) does not hold. Let $\Delta$ be the open triangle

$$
\Delta=\left\{(s, t) \in \mathbb{R}^{2} ;-1<s<t<1\right\}
$$

in the real plane. Consider the real-valued continuous function $F$ on $\Delta$ defined by

$$
F(s, t)=f(t)-f(s) .
$$

Since $f$ is not strictly monotonic, the function $F$ takes both non-negative and non-positive values. It therefore takes the value zero somewhere in the connected space $\Delta$, i. e. there exist $s_{1}$ and $s_{2}$ such that

$$
-1<s_{1}<s_{2}<1, \quad f\left(s_{1}\right)=f\left(s_{2}\right) .
$$

Since $f$ has no local minimum in the open interval $\left(s_{1}, s_{2}\right)$, it follows that $f$ attains its least upper bound in the closed interval $\left[s_{1}, s_{2}\right]$ at an interior point $\xi$ of the interval. Clearly $f$ has a local maximum at $\xi$. Moreover $f$ increases strictly in $(-1, \xi]$. For otherwise we could find $s^{\prime}$ and $s^{\prime \prime}$ such that

$$
-1<s^{\prime}<s^{\prime \prime} \leqq \xi, \quad f\left(s^{\prime}\right) \geqq f\left(s^{\prime \prime}\right) .
$$


The greatest lower bound of $f$ in $\left[s^{\prime}, \xi\right]$ would then be attained at an interior point, at which $f$ would have a local minimum. Similarly $f$ decreases strictly in $[\xi, 1)$, so that condition (b) holds.

Thus we have an open covering $\mathscr{V}$ of $M$ and for each $V$ in $\mathscr{V}$ a $C^{\infty}$ function $s_{V}$ on $V$ which has no critical points and is such that

$$
\sigma \mid V=f \circ s_{V}
$$

where $f$ is a continuous function satisfying either condition (a) or condition (b) above. We remark that if condition (a) holds all points of $V$ are of type (i), while, if condition (b) holds, those points $y$ of $V$ for which $\sigma(y)=\xi$ are of type (ii) and the remaining points of $V$ are of type (i).

It is now clear that our goal will be achieved if we can prove that there are no type (ii) points. For then condition (a) must hold for each $V$ in $\mathscr{V}$. By changing the sign of $s_{V}$ if necessary, we can ensure that $f$ is a strictly increasing function and hence that $s_{V}$ is a $C^{\infty}$ local empirical entropy on $V$. The remainder of this section is therefore devoted to proving that there can be no type (ii) points.

Let $x$ be an arbitrary point of $M, N_{x}$ the mutual accessibility class to which $x$ belongs. We shall show that the points of $N_{x}$ are either all of type (i) or all of type (ii). Let $N_{x}^{(i)}$ be the set of all type (i) points of $N_{x}$ and $N_{x}^{(i i)}$ the set of all type (ii) points of $N_{x}$. Then $N_{x}$ is the disjoint union of $N_{x}^{(i)}$ and $N_{x}^{(i i)}$. But $N_{x}^{(i)}$ and $N_{x}^{(i i)}$ are both open subsets of $N_{x}$. For the family $N_{x} \cap \mathscr{V}$ of all sets of the form $N_{x} \cap V$ for $V \in \mathscr{V}$ is an open covering of $N_{x}$, and each set of $N_{x} \cap \mathscr{V}$ consists either entirely of type (i) points or entirely of type (ii) points. Since $N_{x}$ is connected, by assumption (F), it follows that one or other of the sets $N_{x}^{(i)}$ and $N_{x}^{(i i)}$ must be empty.

Suppose now that $x$ is a point of type (ii). Then, as we have just seen, every point of $N_{x}$ is of type (ii). Consider the non-empty subset $M_{x}$ of $M$ consisting of those points $y$ such that $y \leqq x$. It follows at once from (B) that $M_{x}$ is a closed subset of $M$. But it is also an open subset of $M$. For suppose $y$ belongs to $M_{x}$. Then either $y<x$, in which case it follows from (B) that $y$ has a neighbourhood consisting entirely of points inaccessible from $x$, or $y \sim x$, in which case $y$ is of type (ii) and therefore has a neighbourhood consisting entirely of points from which $y$ and hence $x$ is accessible. Since $M$ is connected, it follows that $M_{x}=M$, i. e. that $y \leqq x$ for all $y$ in $M$. But this contradicts assumption (C). There are therefore no type (ii) points, and our proof is complete.

\section{The Construction of a Differentiable Global Empirical Entropy}

In this section we shall first show that there exists on $(0, \infty)$ a $C^{\infty}$ differentiable structure $\mathscr{D}^{\prime}$, differing in general from the usual one $\mathscr{D}$, such that $\sigma$ is a $C^{\infty}$ submersion of $M$ onto the $C^{\infty}$ differentiable manifold 
$\left\{(0, \infty), \mathscr{D}^{\prime}\right\}$. Since any two $C^{\infty}$ structures on $(0, \infty)$ are equivalent, we shall then be able to construct a $C^{\infty}$ global empirical entropy $s$ on $M$ which has no critical points.

For any set $V$ in $\mathscr{V}$, the set $\sigma(V)$ is an open interval $I$. For it is the image of the open interval $(-1,1)$ under the strictly increasing continuous function $f$ associated with $V$. Since $\mathscr{V}$ is an open covering of $M$, it follows that the set $\mathscr{I}$ of all such intervals $I$ is an open covering of $(0, \infty)$. For each $I$ of $\mathscr{I}$ we pick a $V$ of $\mathscr{V}$ such that $\sigma(V)=I$ and define $s_{I}$ to be the unique local empirical entropy on $\sigma^{-1}(I)$ such that $s_{I} \mid V=s_{V}$. By definition, $s_{I}$ is $C^{\infty}$ on $V$. In fact $s_{I}$ is $C^{\infty}$ on the whole of $\sigma^{-1}(I)$, as we now proceed to show.

Let $t$ be any point of $I$, and let $E$ be the set of all points $x$ of $\sigma^{-1}(t)$ such that $s_{I}$ is $C^{\infty}$ in some neighbourhood of $x$ in $\sigma^{-1}(I)$. Since $V \cap \sigma^{-1}(t)$ $\neq \emptyset, E$ is non-empty, and $E$ is an open subset of $\sigma^{-1}(t)$ by definition. It is also a closed subset of $\sigma^{-1}(t)$.

For suppose $x \in \bar{E}$, and let $W$ be a neighbourhood of $x$ in $\mathscr{V}$. Then, since $s_{I}$ and $s_{W}$ are both local empirical entropies on $W \cap \sigma^{-1}(I)$, it follows that $s_{I}(y)=F\left\{s_{W}(y)\right\}$ for $y$ in $W \cap \sigma^{-1}(I)$, where $F$ is a strictly increasing function on the open interval $s_{W}\left\{W \cap \sigma^{-1}(I)\right\}$. But $x \in \bar{E}$, so that $W$ contains a point $y$ of $E$. Thus $s_{I}$ is $C^{\infty}$ in some neighbourhood of $y$ in $W \cap \sigma^{-1}(I)$. It follows that $F$ is $C^{\infty}$ on an open interval $J$ containing $s_{W}(x)=s_{W}(y)$. Hence $s_{I}$ is $C^{\infty}$ on the open neighbourhood $s_{W}^{-1}(J)$ of $x$ in $\sigma^{-1}(I)$, so that $x \in E$. Thus $E$ is a non-empty open and closed subset of the subspace $\sigma^{-1}(t)$ of $M$. But $\sigma^{-1}(t)$ is a mutual accessibility class and is therefore connected, by assumption (F). Consequently $E=\sigma^{-1}(t)$, i. e. $s_{I}$ is $C^{\infty}$ in the neighbourhood of each point of $\sigma^{-1}(t)$. Since $t$ was an arbitrary point of $I$, it follows that $s_{I}$ is a $C^{\infty}$ function on $\sigma^{-1}(I)$.

Since $s_{I}$ is a $C^{\infty}$ local empirical entropy on $\sigma^{-1}(I)$, it follows that $s_{I}(x)=\varphi_{I}\{\sigma(x)\}$ for $x$ in $\sigma^{-1}(I)$, where $\varphi_{I}$ is a strictly increasing continuous function on $I$. If $I$ and $J$ belong to $\mathscr{I}$ and $I \cap J \neq \emptyset$, then $s_{I}$ and $s_{J}$ are both $C^{\infty}$ on $\sigma^{-1}(I \cap J)$. Consequently $\varphi_{J} \circ\left(\varphi_{I \mid I \cap J}\right)^{-1}$ is a strictly increasing $C^{\infty}$ function on $\varphi_{I}(I \cap J)$. It follows that the family of local charts $\left\{I, \varphi_{I}\right\}_{I \in \mathscr{I}}$ constitutes an atlas for a $C^{\infty}$ differentiable structure $\mathscr{D}^{\prime}$ on $(0, \infty)$, and clearly $\sigma$ is a $C^{\infty}$ map of $M$ onto the $C^{\infty}$ differentiable manifold $\left\{(0, \infty), \mathscr{D}^{\prime}\right\}$.

Next we construct an order preserving diffeomorphism $g$ of $\{(0, \infty)$, $\left.\mathscr{D}^{\prime}\right\}$ onto $\{(0, \infty), \mathscr{D}\}$. Let $\left\{f_{\alpha}\right\}_{\alpha \in A}$ be a $C^{\infty}$ partition of unity [5] on the (paracompact) $C^{\infty}$ differentiable manifold $\left\{(0, \infty), \mathscr{D}^{\prime}\right\}$ which is subordinated to the open covering $\mathscr{I}$ of $(0, \infty)$. For each $\alpha$ in $A$ we choose an interval $I_{\alpha}$ of $\mathscr{I}$ containing the support of $f_{\alpha}$ and write $\varphi_{I_{\alpha}}=\varphi_{\alpha}$. Now $(0, \infty)$ is a 1-dimensional manifold, so that every $C^{\infty}$ differential 1 -form on $\left\{(0, \infty), \mathscr{D}^{\prime}\right\}$ is closed. Furthermore, $(0, \infty)$ is contractible, so 
that every closed 1 -form on $\left\{(0, \infty), \mathscr{D}^{\prime}\right\}$ is exact. Thus every $C^{\infty} 1$-form on $\left\{(0, \infty), \mathscr{D}^{\prime}\right)$ is exact. In particular, there exists a $C^{\infty}$ function $g_{0}$ on $\left\{(0, \infty), \mathscr{D}^{\prime}\right\}$ such that

$$
\sum_{\alpha \in A} f_{\alpha} d \varphi_{\alpha}=d g_{0}
$$

Since each $\varphi_{\alpha}$ is a strictly increasing function, it follows that $g_{0}$ is a strictly increasing function, and clearly $g_{0}$ is a diffeomorphism of $\left\{(0, \infty), \mathscr{D}^{\prime}\right\}$ onto an open interval (possibly infinite) of the real line with its ordinary $C^{\infty}$ structure. Composing $g_{0}$ with any order-preserving diffeomorphism of the latter onto $\{(0, \infty), \mathscr{D}\}$, we obtain a stritly increasing function $g$, which is a $\left(C^{\infty}\right)$ diffeomorphism of $\left\{(0, \infty), \mathscr{D}^{\prime}\right\}$ onto $\{(0, \infty), \mathscr{D}\}$.

Since $g$ is a strictly increasing function it follows that the $C^{\infty}$ map $s$ $=g \circ \sigma$ of $M$ onto $\{(0, \infty), \mathscr{D}\}$ is a $C^{\infty}$ global empirical entropy on $M$. Moreover $s$ has no critical points, i. e. $d s$ never vanishes. For $s$ may be expressed on each set $V$ of the open covering $\mathscr{V}$ of $M$ as a strictly increasing $C^{\infty}$ function of the corresponding $C^{\infty}$ local empirical entropy $s_{V}$, and $d s_{V}$ is everywhere non-zero on $V$. It follows from (E) that $\psi=\lambda d s$, where $\lambda$ is an everywhere non-vanishing $C^{\infty}$ function on $M$ whose reciprocal is thus a global integrating factor for $\psi$.

Acknowledgement. I am indebted to Prof. P. T. LandsBerg for reading a preliminary version of this paper and encouraging me to proceed with it. I also wish to thank Dr. D. BARDEN and Dr. J. P. DovgheRTy for interesting discussions.

\section{Appendix}

We shall here prove the theorem quoted in the introduction. Since it is obvious that (iii) $\Rightarrow$ (i), it will be sufficient to prove that (i) $\Rightarrow$ (ii) and that (ii) $\Rightarrow$ (iii).

Proof of (i) $\Rightarrow$ (ii)

It will be more convenient to prove the equivalent result,

$$
\operatorname{not}(\mathrm{ii}) \Rightarrow \operatorname{not}(\mathrm{i}) \text {. }
$$

Suppose then that (ii) does not hold, and let $x_{0}$ be a point at which $\psi \wedge d \psi \neq 0$. Let $V$ be any open neighbourhood of $x_{0}$ in $M$. We shall show that $x_{0}$ has a neighbourhood $W$ contained in $V$ such that each point of $W$ can be connected to $x_{0}$ by a piece-wise $C^{\infty}$ path $\gamma$ in $V$ which satisfies the condition

$$
\psi\{\dot{\gamma}(t)\}=0 \quad \text { whenever } \quad \dot{\gamma}(t) \text { is defined . }
$$

Since $\psi$ never vanishes, we may suppose without loss of generality that a local coordinate system $\left(x_{1}, \ldots, x_{n}\right)$ is defined on $V$ in which $x_{0}$ is represented by the origin $(0, \ldots, 0)$ of $\mathbb{R}^{n}$ and $\psi$ takes the form

$$
\psi=\sum_{i=1}^{n} a_{i}\left(x_{1}, \ldots, x_{n}\right) d x_{i}
$$


on $V$ where

$$
\begin{gathered}
a_{i}(0, \ldots, 0)=0 \text { for } i<n \\
a_{n}(0, \ldots, 0)=1
\end{gathered}
$$

and $a_{n}\left(x_{1}, \ldots, x_{n}\right)$ is (strictly) positive throughout its domain of definition, so that we can define $C^{\infty}$ functions $b_{i}\left(x_{1}, \ldots, x_{n}\right)$ for $i<n$ by

$$
b_{i}\left(x_{1}, \ldots, x_{n}\right)=-a_{i}\left(x_{1}, \ldots, x_{n}\right)\left\{a_{n}\left(x_{1}, \ldots, x_{n}\right)\right\}^{-1} \text {. }
$$

The above local coordinate system on $V$ will be adhered to throughout this part of the proof, and we shall not always be completely scrupulous in distinguishing between points of $V$ and their representatives in $\mathbb{R}^{n}$ with respect to this coordinate system.

Let $\varphi_{1}(t), \ldots, \varphi_{n-1}(t)$ be arbitrary $C^{\infty}$ real-valued functions on the closed unit interval $0 \leqq t \leqq 1$ such that

$$
\varphi_{i}(0)=\varphi_{i}(1)=0 \quad(i=1, \ldots, n-1) .
$$

Then we can find positive quantities $\eta, \delta$ and $C$ satisfying $\eta C \leqq \delta$ such that the $C^{10}$ function

$$
f(\lambda, t, u)=\sum_{i<n} b_{i}\left\{\lambda \varphi_{1}(t), \ldots, \lambda \varphi_{n-1}(t), u\right\} \dot{\varphi}_{i}(t)
$$

of the three real variables $\lambda, t$ and $u$ is well-defined and bounded above in absolute value by $C$ when $|\lambda| \leqq \eta, 0 \leqq t \leqq 1$ and $|u| \leqq \delta$. Since $f$ is differentiable, there exists a positive constant $K$ such that $f$ satisfies the Lipschitz condition

for

$$
\left|f\left(\lambda, t, u_{1}\right)-f\left(\lambda, t, u_{2}\right)\right| \leqq K\left|u_{1}-u_{2}\right|
$$

$$
|\lambda| \leqq \eta, \quad 0 \leqq t \leqq 1, \quad\left|u_{1}\right| \leqq \delta, \quad\left|u_{2}\right| \leqq \delta .
$$

Let $Y$ be the (real) Banach space [10] of all continuous real-valued functions $y$ on the compact space $[-\eta, \eta] \times[0,1]$ under the supremum norm

$$
\|y\|=\sup \{|y(\lambda, t)| ;|\lambda| \leqq \eta, 0 \leqq t \leqq 1\}
$$

and let $B_{\delta}$ be the closed ball of radius $\delta$ centre 0 in $Y$. Then $B_{\delta}$ is a complete metric space with respect to the metric

$$
d\left(y_{1}, y_{2}\right)=\left\|y_{1}-y_{2}\right\| \text {. }
$$

Let $T$ be the (generally non-linear) mapping of $B_{\delta}$ into itself defined by

$$
(T y)(\lambda, t)=\lambda \int_{0}^{t} f\{\lambda, \tau, y(\lambda, \tau)\} d \tau
$$

Then, since $f$ satisfies the Lipschitz condition (5), it follows that for any two functions $y_{1}$ and $y_{2}$ in $B_{\delta}$ we have

$$
\left|\left(T y_{1}\right)(\lambda, t)-\left(T y_{2}\right)(\lambda, t)\right| \leqq \eta K t\left\|y_{1}-y_{2}\right\|
$$


for $|\lambda| \leqq \eta, 0 \leqq t \leqq 1$. Arguing inductively, we find that

$$
\left\|T^{m} y_{1}-T^{m} y_{2}\right\| \leqq \frac{(\eta K)^{m}}{m !}\left\|y_{1}-y_{2}\right\|
$$

Thus, if $m$ is large enough to make

$$
\frac{(\eta K)^{m}}{m !}<1
$$

then $T^{m}$ is a contraction mapping [10] of the complete metric space $B_{\delta}$ into itself. It follows [10] that $T$ has a unique fixed point $y$ in $B_{\delta}$. The function $y$ satisfies the integral equation

$$
y(\lambda, t)=\lambda \int_{0}^{t} f\{\lambda, \tau, y(\lambda, \tau)\} d \tau
$$

It therefore depends differentiably on $t$ and satisfies the differential equation

with initial condition

$$
\frac{\partial y(\lambda, t)}{\partial t}=\lambda f\{\lambda, t, y(\lambda, t)\}
$$

$$
y(\lambda, 0)=0 .
$$

Recalling the definitions (2) and (4), we see that the $C^{\infty}$ path $\gamma$ in $V$ which is represented in the given local coordinate system by the $n C^{\infty}$ functions

$$
\begin{aligned}
x_{1}(t) & =\lambda \varphi_{1}(t) \\
\vdots & \\
x_{n-1}(t) & =\lambda \varphi_{n-1}(t) \\
x_{n}(t) & =y(\lambda, t)
\end{aligned}
$$

on the closed unit interval satisfies condition (1). Its initial point $\gamma(0)$ is $x_{0}$ and its final point $\gamma(1)$ has coordinates $\{0, \ldots, 0, y(\lambda, 1)\}$. Our next step will be to show that by appropriate choice of the arbitrary functions $\varphi_{i}$ and the real parameter $\lambda$ we can give $y(\lambda, 1)$ any desired real value in a certain neighbourhood of zero.

It follows at once from (6) that $y(0, t)=0$ and that

$$
\lambda^{-1} y(\lambda, t) \rightarrow 0 \text { as } \lambda \rightarrow 0
$$

uniformly in $t$ for $0 \leqq t \leqq 1$. Substituting the definition (4) into (6) and noting that the functions $b_{i}$ are differentiable, we find that

where

$$
\lambda^{-2} y(\lambda, 1) \rightarrow \sum_{i, j<n} b_{i, j}(0, \ldots, 0) \int_{0}^{1} \dot{\varphi}_{i}(t) \varphi_{j}(t) d t \quad \text { as } \quad \lambda \rightarrow 0,
$$

$$
b_{i, j}\left(x_{1}, \ldots, x_{n}\right)=\frac{\partial b_{i}\left(x_{1}, \ldots, x_{n}\right)}{\partial x_{j}} .
$$


Partial integration and use of (3) allow us to rewrite this in the form

$$
\lim _{\lambda \rightarrow 0} \lambda^{-2} y(\lambda, 1)=\sum_{1 \leqq i<j \leqq n-1}\left\{b_{i, j}(0, \ldots, 0)-b_{j, i}(0, \ldots, 0)\right\} \int_{0}^{1} \dot{\varphi}_{i}(t) \varphi_{j}(t) d t .
$$

Now at the point $x_{0}$ we have

$$
\psi \wedge d \psi=d x_{n} \wedge \sum_{i, j<n} b_{i, j}(0, \ldots, 0) d x_{i} \wedge d x_{j} \neq 0 .
$$

Therefore at least one of the quantities $b_{i, j}(0, \ldots, 0)-b_{j, i}(0, \ldots, 0)$ for $1 \leqq i<j \leqq n-1$ must be non-zero. By suitable choice of the arbitrary functions $\varphi_{i}$ we can therefore arrange to make the right hand side of equation (7) (strictly) positive. When this is the case, the function $y(\lambda, 1)$ must take a positive value $\varepsilon_{1}$ for some positive value $\lambda_{1}$ of $\lambda$. By continuity, the values attained by this function for $\lambda$ in the range $0 \leqq \lambda \leqq \lambda_{1}$ must include all real numbers between 0 and $\varepsilon_{1}$. Similarly, by a different choice of the functions $\varphi_{i}$, we can make the right hand side of (7) negative. In this case we can find a positive number $\varepsilon_{2}$ such that $y(\lambda, 1)$ can take every value between $-\varepsilon_{2}$ and 0 for suitable $\lambda$. Thus, for each value of $x_{n}$ in the closed interval $\left[-\varepsilon_{2}, \varepsilon_{1}\right]$, the point $\left(0, \ldots, 0, x_{n}\right)$ can be connected to $x_{0}$ by a $C^{\infty}$ path in $V$ satisfying (1).

Now it follows from the existence and uniqueness theorem for solutions of ordinary differential equations [11] that there exist positive numbers $\mu$ and $\sigma$ such that, for all values of the real constants $x_{1}, \ldots, x_{n}$ satisfying $x_{1}^{2}+\cdots+x_{n}^{2}<\mu^{2}$, the differential equation

$$
\frac{d y}{d s}=\sum_{i<n} x_{i} b_{i}\left(s x_{1}, \ldots, s x_{n-1}, y\right)
$$

has a unique solution in the range $0 \leqq s \leqq \sigma$ satisfying the initial condition

$$
y=x_{n} \quad \text { when } \quad s=0 .
$$

Moreover this solution $y\left(s, x_{1}, \ldots, x_{n}\right)$ is a $C^{\infty}$ function of $s$ and of the $n$ real parameters $x_{1}, \ldots, x_{n}$.

Consider the $C^{\infty}$ map $f$ of the open ball $x_{1}^{2}+\cdots+x_{n}^{2}<\mu^{2}$ in $\mathbb{R}^{n}$ into $V$ defined by

$$
f\left(x_{1}, \ldots, x_{n}\right)=\left\{\sigma x_{1}, \ldots, \sigma x_{n-1}, y\left(\sigma, x_{1}, \ldots, x_{n}\right)\right\} .
$$

Since $y\left(\sigma, 0, \ldots, 0, x_{n}\right)=x_{n}$, we see that the Jacobian of $f$ has the positive value $\sigma^{n-1}$ at the origin. Hence there exists a number $\varrho$ satisfying

$$
0<\varrho \leqq \min \left(\varepsilon_{1}, \varepsilon_{2}\right)
$$

such that $f$ maps the open ball $x_{1}^{2}+\cdots+x_{n}^{2}<\varrho^{2}$ in $\mathbb{R}^{n}$ diffeomorphically onto an open neighbourhood $W$ of $x_{0}$ in $V$.

5 Commun. math. Phys., Vol. 10 
Consider now an arbitrary point $f\left(x_{1}, \ldots, x_{n}\right)$ of $W$. Then the $C^{\infty}$ path in $V$ defined by

$$
t \rightarrow\left\{t \sigma x_{1}, \ldots, t \sigma x_{n-1}, y\left(t \sigma, x_{1}, \ldots, x_{n}\right)\right\}
$$

for $0 \leqq t \leqq 1$ has $f\left(x_{1}, \ldots, x_{n}\right)$ as its final point. Since the function $y$ is a solution of the differential equation (8) with initial condition (9), it follows that this path satisfies condition (1) and has initial point $\left(0, \ldots, 0, x_{n}\right)$. But $\left|x_{n}\right|<\varrho$, and therefore in view of $(10)$ this initial point may be connected to $x_{0}$ by a $C^{\infty}$ path in $V$ satisfying (1). Thus each point of $W$ may be connected to $x_{0}$ by a piece-wise $C^{\infty}$ path in $V$ satisfying (1).

Proof of (ii) $\Rightarrow$ (iii)

The proof will be by induction on the dimension $n$ of the manifold $M$. The result holds trivially for 1-dimensional manifolds. We shall suppose that it holds for $(n-1)$-dimensional manifolds, where $n>1$.

Let $M$ be $n$-dimensional, suppose that $\psi$ satisfies (ii), and let $x_{0}$ be an arbitrary point of $M$. Then we can define a local coordinate system $\left(x_{1}, \ldots, x_{n}\right)$ in the neighbourhood of $x_{0}$ such that $x_{0}$ is represented by the origin of $\mathbb{R}^{n}$ and $\psi$ takes the form

$$
\psi=\sum_{i=1}^{n} a_{i}\left(x_{1}, \ldots, x_{n}\right) d x_{i}
$$

where $a_{n-1}(0, \ldots, 0) \neq 0$. By continuity $a_{n-1}$ is non-zero in a neighbourhood of the origin, and, for all sufficiently small values of the real constants $y_{1}, \ldots, y_{n-1}$, the differential equation

$$
\frac{d x_{n-1}}{d y_{n}}=-a_{n}\left(y_{1}, \ldots, y_{n-2}, x_{n-1}, y_{n}\right)\left\{a_{n-1}\left(y_{1}, \ldots, y_{n-2}, x_{n-1}, y_{n}\right)\right\}^{-1}
$$

with initial condition

$$
x_{n-1}=y_{n-1} \text { when } y_{n}=0
$$

has a unique solution (11)

$$
x_{n-1}=F\left(y_{1}, \ldots, y_{n-2}, y_{n-1}, y_{n}\right)
$$

for $y_{n}$ in a certain neighbourhood of zero. Moreover $F\left(y_{1}, \ldots, y_{n}\right)$ is a $C^{\infty}$ function on the neighbourhood of the origin in $\mathbb{R}^{n}$ on which it is defined. Consider the $C^{\infty}$ map

$$
\left(y_{1}, \ldots, y_{n}\right) \rightarrow\left\{y_{1}, \ldots, y_{n-2}, F\left(y_{1}, \ldots, y_{n}\right), y_{n}\right\}
$$

of this neighbourhood into $\mathbb{R}^{n}$. It maps the origin onto itself, and its Jacobian has the value 1 at the origin. It therefore maps an open neighbourhood $U_{y}$ of the origin in $\mathbb{R}^{n}$ diffeomorphically onto another open neighbourhood $U_{x}$ of the origin in $\mathbb{R}^{n}$. On the open neighbourhood $U$ of $x_{0}$ represented in the coordinate system $\left(x_{1}, \ldots, x_{n}\right)$ by $U_{x}$, we can therefore define a new coordinate system $\left(y_{1}, \ldots, y_{n}\right)$ taking values 
in $U_{y}$ by

$$
\begin{aligned}
x_{i} & =y_{i} \text { for } i \neq n-1 \\
x_{n-1} & =F\left(y_{1}, \ldots, y_{n}\right) .
\end{aligned}
$$

In terms of these coordinates on $U, \psi$ takes the form

$$
\psi=\sum_{i=1}^{n-1} b_{i}\left(y_{1}, \ldots, y_{n}\right) d y_{i} .
$$

Now $\psi$ satisfies condition (ii) by hypothesis. In the new coordinate system on $U$, this condition takes the form

$$
\psi \wedge d \psi=-\sum_{1 \leqq i<j \leqq n-1}\left(b_{i} d b_{j}-b_{j} d b_{i}\right) \wedge d y_{i} \wedge d y_{j}=0 .
$$

It follows immediately from (11) that

$$
b_{i} \frac{\partial b_{j}}{d y_{n}}-b_{j} \frac{\partial b_{i}}{\partial y_{n}}=0 .
$$

Since at least one of the $b_{i}$ is non-zero at the origin and hence in a neighbourhood of the origin, we infer from (12) that these functions must be of the form

$$
b_{i}\left(y_{1}, \ldots, y_{n}\right)=h\left(y_{1}, \ldots, y_{n}\right) c_{i}\left(y_{1}, \ldots, y_{n-1}\right)
$$

for all sufficiently small values of $y_{1}, \ldots, y_{n}$, where the function $h$ never vanishes. Substituting (13) into (11), we find that the differential 1-form

$$
\omega=\sum_{i=1}^{n-1} c_{i}\left(y_{1}, \ldots, y_{n-1}\right) d y_{i}
$$

on an open subset of $\mathbb{R}^{n-1}$ satisfies

$$
\omega \wedge d \omega=0 \text {. }
$$

Since $\omega$ never vanishes it follows by the induction hypothesis that $\omega$ is of the form

$$
\omega=\lambda d g
$$

in some open neighbourhood of the origin in $\mathbb{R}^{n-1}$. Hence

$$
\psi=f d g
$$

in some open neighbourhood $V$ of $x_{0}$ in $M$, where $g$ is the function of the local coordinates $y_{1}, \ldots, y_{n-1}$ that appears in (14), and

$$
f\left(y_{1}, \ldots, y_{n}\right)=h\left(y_{1}, \ldots, y_{n}\right) \lambda\left(y_{1}, \ldots, y_{n-1}\right) \text {. }
$$

\section{References}

1. Bernstein, B.: J. Math. Phys. 1, 222 (1960).

2. Carathéodory, C.: Math. Ann. 67, 355 (1909).

3. BuchdahL, H. A.: The concepts of classical thermodynamics. Cambridge: University Press 1966. 
4. Landsberg, P. T.: Thermodynamics. New York: Interscience 1961.

5. De Rham, G.: Variétés Différentiables. Paris: Hermann 1960. Singer, I. M., and J. A. THORPe: Lecture notes on elementary topology and geometry. Glenview, Ill.: Scott and Foresman 1967.

6. Buchdahl, H. A., and W. Greve: Z. Physik 168, 386 (1962).

7. Landsberg, P. T.: Phys. Stat. Sol. 1, 120 (1961).

8. Kowalski, H. J.: Topological spaces. New York: Academic Press 1964.

9. Nachbin, L.: Topology and order. Princeton, N. J.: Van Nostrand 1965.

10. Kolmogorov, A. N., and S. V. Fomin: Elements of the theory of functions and functional analysis. Vol. I. Rochester, N. Y.: Graylock 1957.

11. Murray, F. J., and K. S. Mrruer: Existence theorems for ordinary differential equations. New York: University Press 1954.

\author{
J. B. Boyling \\ Department of Applied \\ Mathematics and Theoretical Physics \\ University of Cambridge \\ Cambridge, Great Britain
}

